
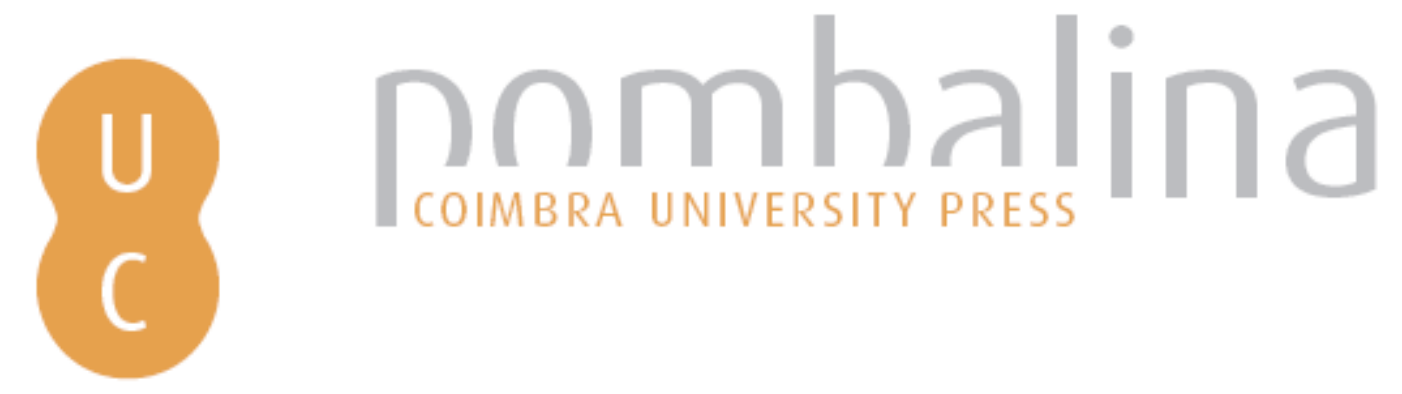

\title{
Equipas de trabalho: eficácia ou eficácias?
}

Autor(es): $\quad$ Lourenço, P.R.; Miguez, J.; Gomes, A. D.; Freire, P.

Publicado por: Imprensa da Universidade de Coimbra

URL persistente:

URI:http://hdl.handle.net/10316.2/32742

DOI:

DOl:http://dx.doi.org/10.14195/978-989-26-0452-7_4

Accessed : $\quad$ 26-Apr-2023 15:14:05

A navegação consulta e descarregamento dos títulos inseridos nas Bibliotecas Digitais UC Digitalis, UC Pombalina e UC Impactum, pressupõem a aceitação plena e sem reservas dos Termos e Condições de Uso destas Bibliotecas Digitais, disponíveis em https://digitalis.uc.pt/pt-pt/termos.

Conforme exposto nos referidos Termos e Condições de Uso, o descarregamento de títulos de acesso restrito requer uma licença válida de autorização devendo o utilizador aceder ao(s) documento(s) a partir de um endereço de IP da instituição detentora da supramencionada licença.

Ao utilizador é apenas permitido o descarregamento para uso pessoal, pelo que o emprego do(s) título(s) descarregado(s) para outro fim, designadamente comercial, carece de autorização do respetivo autor ou editor da obra.

Na medida em que todas as obras da UC Digitalis se encontram protegidas pelo Código do Direito de Autor e Direitos Conexos e demais legislação aplicável, toda a cópia, parcial ou total, deste documento, nos casos em que é legalmente admitida, deverá conter ou fazer-se acompanhar por este aviso. 


\section{A. DUARTE GOMES - ANTÓNIO CAETANO JOSÉ KEATING • MIGUEL PINA E CUNHA

\author{
Coordenadores
}

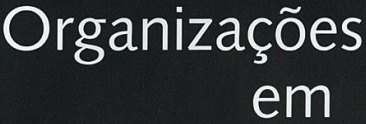

Transição

Contributo da Psicologia do Trabalho

e das Organizaçōes 
(Página deixada propositadamente em branco) 


\section{A. DUARTE GOMES - JOSÉ KEATING ANTÓNIO CAETANO - MIGUEL PINA E CUNHA Coordenadores}

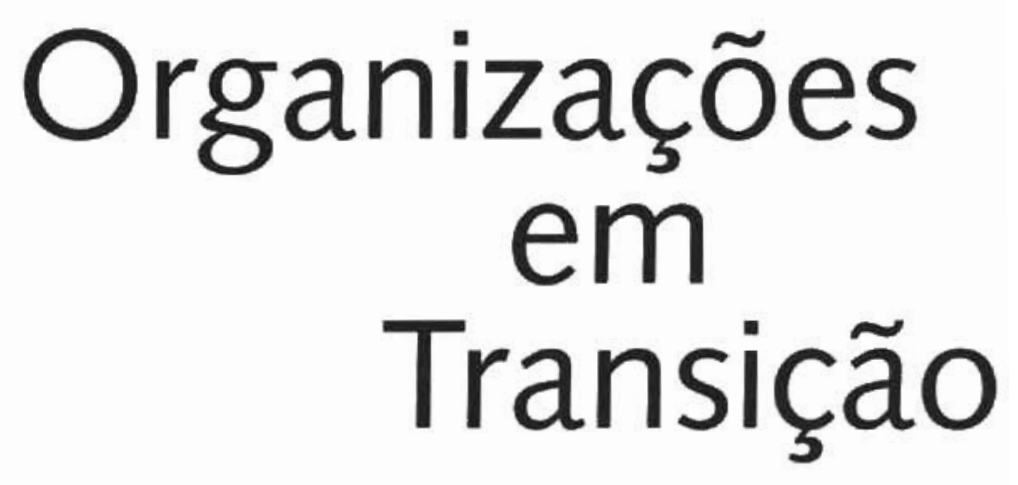

Contributos da Psicologia do Trabalho e das Organizações 


\section{COORDENACÁO EDITORIAL \\ Imprensa da Universidade de Coimbra \\ CONCEPÇÃO GRAFACA \\ António Barros \\ EXECUÇÃO GRAFICA \\ G.C. - Gráfica de Coimbra, Lda. \\ Palheira - Assafarge - Apart. 3068 \\ 3001-453 Coimbra Codex}

ISBN

972-98225-3-0

DEPOSITO LEGAL.

153435/00

(c) JunHo 2000. Imprensa da Universidade de Coimbra 


\title{
EQUIPAS DE TRABALHO: EFICÁCIA OU EFICÁCIAS?
}

\author{
Lourenço, P. R. Miguez, J., Gomes, A. D. \& Freire, P. \\ UNIVERSIDADE DE COIMBRA - FPCEIISR \\ UNIVERSIDADE DO PORTO - FPCE
}

Muitos investigadores apontam os estudos de Hawthorne, iniciados nos anos 20, por Elton Mayo e seus colaboradores (Mayo, 1932), como o primeiro esforço sistemático da ciência para trazer à luz do dia a importância dos grupos nas organizações. Embora inicialmente projectados e desenvolvidos numa perspectiva clássica do trabalho e das organizações e com objectivos situados a nível individual, estes estudos passaram a constituir uma referência na literatura sobre grupos e equipas de trabalho, na medida em que as observaçōes então efectuadas, ao longo dos cinco anos em que decorreram as investigações, conduziram a que, de forma surpreendente para a própria equipa de investigadores, o nível grupal se tenha tornado o principal alvo de análise. Desde então, e até aos nossos dias, o interesse pelo estudo dos grupos, bem como a perspectiva com que são abordados, têm sido alvo de alterações significativas. Se os anos 50 , em que a maior parte das investigações enfatizava os processos de interaç̧ão entre os membros de um grupo e o impacto desta dinâmica nos mesmos (Shea \& Guzzo, 1987; Guzzo \& Shea, 1992), foram dos mais produtivos para o estudo dos grupos, a década de 60 . marcada pelo triunfo do indivíduo como unidade de análise das Ciências Sociais (Graumann, 1986; Farr, 1990) conduziu a um forte decréscimo da investigação neste domínio. Se os anos 70 - em particular com os trabalhos de Steiner (1972) e de Hackman \& Morris (1975) visando compreender as rela- 
ções entre os processos grupais e o seu desempenho, assim como os estudos associados ao Movimento da Qualidade de Vida no Trabalho, realizados com base na introdução de equipas no lugar da tradicional linha de produção (v.g. Gyllenhammar, 1977) - constituíram um marco decisivo, ao conduzirem à emergência do interesse pelo estudo dos grupos como entidades desempenhando tarefas (unidades performantes), somente a partir dos anos 80 , com os esforços para a criação de equipas de elevado desempenho se tornou visível uma clara expansão do interesse pelo estudo das equipas de trabalho (team working) e se tornou dominante uma linha de investigação centrada sobre a compreensão das equipas nos seus contextos e nas suas múltiplas facetas. Com o crescente envolvimento da psicologia das organizações na investigação mais recente sobre grupos e/ou equipas de trabalho (em detrimento de outras áreas da psicologia, designadamente da psicologia social, que durante décadas lideraram a investigação nesta temática) esta tendência acentuou-se, tendo conduzido a que a eficácia se tenha tornado central no universo da investigação neste domínio. Uma parte substancial da actual investigação dedicada a grupos e equipas de trabalho incide sobre a temática da performance, desempenho ou eficácia.

Para um olhar menos atento, a reduzida discussão em torno do que se entende por eficácia de um grupo e/ou equipa de trabalho poderá sugerir a sua objectividade, clareza e univocidade. A multiplicidade de estudos, mas sobretudo a diversidade de perspectivas e de modelos que a procuram descrever, explicar e avaliar, apontam, no entanto, em sentido contrário e atestam o reduzido acordo existente no seio da comunidade científica quanto ao seu significado(I). Cada investigador, em função do seu posicionamento teórico-conceptual propõe, na sua linguagem própria, uma diferente forma de identificar, descrever e explicar a eficácia e utiliza distintos critérios e instrumentos de medida para a avaliar (Goodman et al., 1987; Hackman, 1987; Morin et al., 1994).

A revisão da investigação empírica, bem como dos mais representativos modelos explicativos da eficácia dos grupos e/ou equipas de trabalho (geralmente conhecidos como modelos de funcionamento ou de eficácia dos grupos e/ou equipas de trabalho). permite, com efeito, verificar que a eficácia tende a ser vista, umas vezes, como realização de objectivos, produtividade. rendibilidade, desempenho, eficiência ou rendimento (e.g. Nieva et al., 1978;

(1) A semelhança, de resto, do que acontece quando a análise se situa no nivel organizacional. 
Tziner \& Vardi., 1982; Shea \& Guzzo, 1984; Gladstein, 1984; Goodman, 1986; Sundstrom et al., 1990; Tannenbaum et al., 1992), outras, como sobrevivência ou viabilidade de uma equipa de trabalho (e.g. Hackman, 1983; 1987; Gladstein. 1984; Sundstrom et al., 1990), outras ainda, como satisfação dos membros da equipa, sua qualidade de vida, ou intensidade da sua experiência afectiva (e.g. Hackman, 1983; 1987; Sundstrom et al., 1990); por último, numa perspectiva que enfatiza critérios centrados sobre as relações da equipa com o exterior, aparece associada, por exemplo, à satisfação dos clientes (e.g Hackman, 1983, 1987; Middlemist \& Hitt, 1981).

A diversidade assinalada reflecte a coexistência de múltiplas representações de eficácia. Os modelos construídos com base nessas representações fazem sobressair aspectos parciais do problema e não dão conta de todas as contingências nem se adequam a todos os contextos, tornando, deste modo, visiveis os limites do conhecimento científico neste domínio, quer a nível teórico, quer a nível da investigação empírica.

Ao dificultar a delimitação do significado da eficácia e ao sugerir a presença, não de uma, mas de várias eficácias, a pluralidade de concepções impossibilita o desenvolvimento de uma teoria ou modelo geral da eficácia dos grupos e/ou equipas de trabalho. Este facto tem levado a que diversos autores argumentem que, dada a ausência de uma teoria consolidada, a investigação neste domínio mais não é do que um corpo de observações empíricas sem potencial para gerar novos conhecimentos (e.g. Hannan \& Freeman, 1977). Afirmam mesmo que a eficácia não pode ser perspectivada à luz de um conceito científico e propõem o abandono do seu estudo no seio da literatura organizacional.

Em nossa opinião, e na linha seguida por Fernandez-Ríos \& Sanchez (1997), não parece desejável nem justificável o abandono do estudo da eficácia no contexto das organizações e dos grupos e/ou equipas de trabalho. Pelo menos quatro razões podem ser aduzidas a favor do prosseguimento da sua investigação.

Em primeiro lugar, porque os estudos sobre eficácia são centrais para o pensamento organizacional (Goodman \& Pennings, 1977; Pfeffer, 1977; Cameron \& Whetten, 1983). Sob o ponto de vista teórico-conceptual, em todas as abordagens sobre a natureza das organizaçōes se encontram incluídas, implícita ou explicitamente, noções sobre a eficácia e as diferenças que existem entre organizações eficazes e organizações ineficazes. 
Em segundo lugar, porque a necessidade de verificar em que medida um sistema realiza a sua missão, ou a realiza melhor do que outros, conduz a que a noção de eficácia constitua uma questão empírica central.

Em terceiro lugar, porque se trata de uma problemática socialmente relevante. Na medida em que as pessoas emitem continuamente juízos e avaliam o desempenho de cada organização e de cada equipa, a questão da avaliação da eficácia é uma inevitabilidade no quotidiano das organizações e dos grupos e/ou equipas de trabalho.

Em quarto lugar e, last but not the least, porque a ausência de consenso relativo ao significado da eficácia, a inexistência de uma teoria geral e a presença de múltiplas representações, ao contrário de serem um obstáculo à investigação, constituem um desafio à investigação. É esta multiplicidade de representações que ao evidenciar a subjectividade da eficácia permite uma nova inteligibilidade sobre a sua própría noção. A eficácia de um grupo e/ou equipa de trabalho não existe enquanto tal, não é uma realidade objectiva, mas uma construção dessa mesma realidade. É um juízo valorativo, uma avaliação que um indivíduo (avaliador) efectua a respeito de um grupo e/ou equipa de trabalho, nomeadamente no que se refere às actividades, aos produtos e/ou aos resultados e/ou seus efeitos, com base na sua própria representação de eficácia, nos seus valores, interesses e preferências (Beaudin \& Savoie, 1995). Neste sentido, afirmar que uma equipa é eficaz é afirmar que, perante um conjunto de critérios seleccionados pelo avaliador, aquilo que a equipa oferece e a que ele atribui importância, corresponde às suas expectativas.

É porque a eficácia de um grupo ou equipa de trabalho é uma representação que um actor estratégico, activo e construtivo faz dessa mesma realidade - um constructo(2) fundado e integrando os valores de cada um (Quinn \& Rohrbaugh, 1983; Fernandez-Ríos \& Sanchez, 1997) - que a mesma assume, como referem Morin et al. (1994), a especificidade e o particularismo de diferentes definições e, simultaneamente, se torna indeterminada e não passivel de um significado unívoco, consensual, aceite por todos.

(2) Os constructos não são a realidade objectiva. Eles constituem abstraç̧ōes resultantes da acção de observação e análise sobre essa mesma realidade e permitem atribuir-the um sentido (Cameron, 1981). Não faz parte da sua natureza a aceitabilidade por todos. O grau de acertabilidade e legitimidade de que gozam remete para a comunidade no interior da qual eles são gerados e/ou integrados. 
Nesta perspectiva, a coexistência do que parecem ser distintas eficácias (noutros termos, a pluralidade de concepções sobre eficácia), bem como a presença de diferentes modelos de eficácia dos grupos e/ou equipas de trabalho, contribuem para a clarificação conceptual e para o progresso da investigação neste domínio.

Estaremos perante diferentes realidades ou diferentes facetas de uma mesma realidade? Tratar-se-á de diversidade ou de multidimensionalidade? E, nesse caso, haverá alguma forma de organizar as múltiplas dimensões e de as integrar num todo coerente e capaz de permitir um olhar mais abrangente. ainda que necessariamente sempre incompleto, sobre o constructo eficácia?

Com o objectivo de reenquadrar as informações acumuladas nos últimos anos tendo em vista facilitar a intervenção e reorientar a investigação sobre as equipas de trabalho em contexto organizacional, Savoie \& Beaudin (1995) realizaram uma extensa revisão da literatura, quer teórica quer empírica, em que procuraram reagrupar os diferentes critérios de eficácia utilizados pelos investigadores. Nas suas análises, os referidos autores assinalam que as diferentes representações de eficácia dos grupos e/ou equipas de trabalho fazem sobressair um quadro que, com alguma consistência, dá forma à emergência de um constructo multidimensional, composto por quatro dimensões potenciais(3): a) Social (qualidade da experiência grupal); b) Económica (rendimento do grupo); c) Política (legitimidade ou reputação do grupo face à sua constelação): d) Sistémica (perenidade). A dimensão social está intimamente relacionada com a coesão social e diz respeito ao valor dos recursos humanos, no que se refere a aspectos como a qualidade de vida no trabalho, a satisfação, o moral, o desenvolvimento e a mobilização dos seus membros. A dimensão económica está relacionada com a eficiência e a produtividade e pressupõe uma optimização de recursos tendo em vista o alcance dos objectivos a que as organizações e/ou as equipas se propõem. A dimensão política da eficácia diz respeito à avaliação exterior e/ou superior do grupo. Trata-se de uma dimensão que se centra em critérios relacionados com a reputação e a legitimidade da acção de cada equipa de trabalho. Por último, a dimensão sistémica

(3) Potenciais porque, se bem que presentes no universo dos resultados da equipa. os observadores, na maior parte dos casos, não as tomam todas em consideração (ou porque não querem ou porque não podem) nas suas avaliações e/ou nas suas concepçōes implicitas de eficácia. Somente quando tomados pelos avaliadores como critérios para avaliação da eficácia deixam a sua condiçăo potencial e se realizam enquanto critérios de eficácia. 
está relacionada com a perenidade, crescimento, adaptação e estabilidade do sistema-grupo ao longo do tempo e face às mudanças ocorridas na envolvente.

Um olhar atento sobre os diversos modelos e teorias permite constatar a relevância teórica e o potencial de investigação da perspectiva quadridimensional proposta por Savoie \& Beaudin (1995).

Bales (1950) considera a eficácia sob o aspecto satisfação dos membros. De igual modo, entre outros investigadores, Trist \& Bamforth (1951). Hackman \& Oldham (1980), assim como Hackman (1987), ou ainda Sundstrom et al. (1990), integram a satisfação como um dos critérios de avaliação da eficácia de um grupo ou equipa de trabalho. Outros investigadores utilizam também indicadores relacionados com a dimensão social da eficácia, a qual pode ser explicitada nos termos que Hackman (1990) utilizou, como o grau em que a experiência de grupo contribui para o bem-estar e o crescimento pessoal dos membros (grupo/equipa como local de aprendizagem, de apoio afectivo, cooperação, segurança, desenvolvimento pessoal e profissional,...).

O rendimento surge, também, como uma das dimensões mais frequentemente referidas(4). Por exemplo, Steiner (1972) utiliza o critério produtividade, Tziner \& Vardi (1982), Hackman \& Oldham (1980), Sundstrom et al. (1990), assim como a grande maioria dos investigadores no domínio das organizações, fazem referência a critérios passíveis de ser inseridas na dimensão rendimento.

A dimensão legitimidade/reputação, embora menos claramente operacionalizada, está igualmente presente na literatura da especialidade (Savoie \& Beaudin, 1995). Hackman (1990), em particular, precisa esta dimensão quando se refere à avaliação subjectiva da eficácia, proveniente de actores externos à equipa de trabalho. As abordagens que se debruçam sobre os diferentes interessados ou "constituintes múltiplos" ("multiple constituencies") acentuam, igualmente, esta dimensão ao considerarem que a avaliação da eficácia de uma equipa deve ter em conta os critérios e as avaliações efectuadas pela 82 constelação em que a equipa ou grupo de trabalho se insere.

Quanto à dimensão perenidade/viabilidade da equipa, associada à temporalidade, diversos são também os investigadores que a ela se referem e os

(4) Refira-se que, não raras vezes, a eficácia é concebida somente nesta perspectiva. De algum modo, pode mesmo afirmar-se que a perspectiva tradicional da eficácia se reduz ou privilegia esta dimensão. 
modelos que a integram. Assim, Sundstrom et al. (1990) enfatizam o desejo de os membros continuarem a trabalhar em conjunto, Shea \& Guzzo (1984) a capacidade de a equipa desempenhar eficazmente no futuro e Hackman (1990), para citar somente alguns exemplos, acentua o critério relativo ao grau em que a realização das tarefas da equipa eleva a probabilidade de os membros terem desejo de trabalhar em conjunto no futuro.

A revisão da literatura empírica sobre a temática da eficácia realizada por Savoie \& Beaudin (1995) (feita com base em investigações que utilizam indicadores de eficácia sob a forma de auto-descrições de resultados ou do vivido grupal expressos pelos membros, índices externos, do tipo resultados provenientes de registos de produção ou do tipo avaliações sobre resultados observados, feitas por actores externos à equipa ou grupo de trabalho), não sendo, naturalmente, exaustiva, parece dar suporte à sua proposta quadridimensional da eficácia(5).

Importa destacar que das quatro dimensōes identificadas, duas dominam o universo de medida, em termos de critérios e indicadores - Social e Rendimento. As dimensões Legitimidade e Perenidade são aquelas em relação às quais as investigaçōes menos têm centrado a sua atenção(6).

(5) A este propósito os referidos autores afirmam que, se é possível identificar e dar suporte teórico-conceptual às quatro dimensões propostas, elas não são independentes entre si e não representam categorias discretas. Com efeito, na realidade, as fronteiras não são simples de identificar/desenhar, já que as dimensōes são interrelacionadas e, por exemplo, alguns indicadores de uma dimensão podem ser determinantes de indicadores de outra(s) dimen. são(ōes).

(6) Eis algumas das razöes que poderäo estar associadas a este facto: historicamente a investigação sobre grupos for marcada pela psicologia social; a sua incidência no estudo, em laboratóno, das interaç̧ōes no seio do grupo é conhecida. A dimensão social era, então. central e, por contraste, as dimensōes legitimidade (muito associada aos contextos dos grupos) e perenidade (relevante para grupos reais/naturais) completamente marginais ou mesmo ignoradas. Por outro lado, a dimensão social é relativamente fácil de medir (grande parte dos indicadores podem ser avaliados através de auto-descriçōes dos membros da equipa) e existem inúmeros instrumentos concebidos para o efeito. Paralelamente, a estreita ligaçăo entre a Psicologia do Trabalho e das Organizações e a Gestão favoreceram a predominância das medidas de rendimento, as quais sāo muitas vezes mecanizadas ou informatizadas e, entāo, de fácil recolha/acesso (Weick, 1977). Apesar de a perenidade e a legitimidade incluirem indicadores igualmente importantes, terem no domínio organizacional (no estudo dos grupos nos seus contextos) um excelente campo de aplicação e beneficiarem de suporte conceptual tão claro como as duas dimensões anteriores, a sua sub-utilização parece evidente (Savoie \& Beaudin, 1995). 
A perspectiva que temos vindo a explicitar - que, como realçámos, assenta no reconhecimento da eficácia como um constructo de natureza intersubjectiva e, por isso mesmo, causa de variedade e divergência quanto ao significado da eficácia quer entre investigadores quer entre os diversos actores dos sistemas alvo de investigação, quer mesmo entre estes e os investigadores - coloca problemas de certa complexidade à investigação de campo.

Dada a natureza política da eficácia, questões como quem determina os critérios de eficácia, quem decide que medidas devem ser utilizadas ou com base em que modelo conceptual se definirão tais medidas, são de difíil resposta. Na sequência de autores como Quinn \& Rohrbaugh (1983), Cameron \& Whetten (1985). Morin et al. (1994) e Fernandez-Ríos \& Sanchez (1997), para quem a eficácia (enquanto realidade subjectiva) é indissociável dos actores e dos seus contextos (isto é, não é "context-free"), somos de opinião que na investigaçāo empírica sobre avaliação da eficácia, mais do que oferecer critérios, é preciso analisar que critérios os actores do sistema-alvo consideram indicadores válidos de eficácia. Mais do que centrada sobre as representações de eficácia dos investigadores, os estudos sobre eficácia devem apoiar-se numa perspectiva intersubjectiva, multifacetada e contingencial, centrada nos valores e representações existentes no sistema em que se insere a equipa. $\mathrm{Na}$ nossa perspectiva, e na linha de pensamento das chamadas abordagens de múltiplos constituintes (e.g. Connoly et al. 1980; Cummings, 1981; Goodman, 1986) ela deve ter em consideração os valores dos diferentes grupos que interagem com a equipa que está a ser objecto de investigação (por exemplo os membros da a equipa, o seu superior, outras equipas da organização, os clientes, o topo estratégico...). O significado da eficácia e os critérios da sua avaliação estarão, assim, conectados com a realidade específica a investigar.

Terminamos retomando a pergunta que serviu de ponto de partida e título para o presente artigo: com base nas principais ideias e reflexões que efectuámos, tal questão - "eficácia ou eficócias?" - parece ainda pertinente?

84 Em nosso entender, ela constitui somente o reflexo de um falso problema que assenta num posicionamento dicotómico que não admite alternativas: ou existe acordo sobre o significado da eficácia, existe uma realidade - a eficácia - e uma teoria geral da eficácia ou, por contraste, existem diferentes realidades - diferentes eficácios - incompatíveis e sem possibilidade de integração.

Somos de opinião que existe uma perspectiva alternativa que admite e considera mesmo desejável a coexistência da utilização dos dois termos - 
eficácia e eficácios - na medida em que se trata de uma mesma realidade, capaz de ser identificada por um mesmo vocábulo que remete para um mesmo constructo - eficácia -, o qual assume diversas facetas - eficácias que mais não são do que a sua operacionalização intersubjectiva, em cada contexto e por cada actor, feita a partir das múltiplas dimensões potenciais que o constituem.

Dito de outra forma, se por um lado, a eficácia não pode ser avaliada nem completamente entendida utilizando apenas um modelo ou um ponto de vista, por outro lado, a investigação não pode deixar de ter presente que os diferentes modelos e suas representaçōes da eficácia constituem somente diversas faces de uma mesma realidade.

\section{REFERÊNCIAS}

BALES, R. F. (1950), A Set of Categories for the Analysis of Small Group Interaction. American Sociological Review, 15, 257-263

BEAUDIN, G. \& SAVOIE, A. (1995). L'éfficacité des équipes de travail: definitions, composants et mesures. Revue Québecoise de Psychologie, I16, (1), 185-201.

CAMERON. K. S. (198I). Domains of Organizational Effectiveness in Colleges and Universities. Management Joumal, 24, 25-47

CAMERON. K. S. \& WHETTEN. D. A. (1983). Organizational Effectiveness: a Comparison of Multiple Models.Academic Press Inc.

CONNOLLY.T.A., CONLON, E. J. \& DEUTSCH. S. J. (1980). Organizational Effectiveness: a Multiple Constituency Approach. Academy of Management Review, 5, 211-218

CUMMINGS, T. (198I). Designing effective Work Groups, In P. C. Nystrom III and W. H. Starbuck (eds.), Handbook of Organizational Design: Vol, 2 Remodeling organization and their environments. Oxford, England: Oxford University Press.

FARR, R (1990). Waxing and waning of interest in societal psychology, historical perspective.In H. Himmelweit \& G. Gaskell (eds) Societal Psychology. Newbury Park, CA: Sage.

FERNÁNDEZ-RIOS \& SÁNCHEZ, M. (1997). Eficacia Organizacional - Concepto, Desarrollo y Evaluoción. Madrid: Diaz de Santos.

GLADSTEIN, D. (1984). A model of task group effectiveness. Administrative Science Quarteriy, 29 (4), 499-517.

GOODMAN, P.S. (1986). Designing Effective Work Groups. San Francisco: Jossey-Bass.

GOODMAN, P. S. \& PENNINGS, J. M. (1977). New Perspectives on Organizational Effectiveness. San Francisco: Jossey-Bass.

GOODMAN, P., RAVLIN, E. \& SCHMINKE, M. (1987). Understanding Groups in Organizations. Research in Organizational Behovior, 9, 121-173.

GRAUMANN, C. F. (1986). The individualization of the social and desocialization of the individual: Floyd H. Allport's contribution to social psychology. In C. F. Graumann \& S. Moscovici (eds). Changing Conceptions of Crowd Mind and Behavior. New York Springer-Verlag. 
GUZ7O, R \& SHEA, G. (1992). Group Performance and Intergroup Relations in Organizations In M.. D. Dunnette, \& L. M. Hough (eds.). Handbook of Industrial and Organizational Psychology. 3, Palo Alto, CA: Consulting Psychologists Press.

GYLENHAMMAR, P. G. (1977). How Volvo Adapts Work to People. Harvard Business Review. July-August. 102-113.

HACKMAN, J. R. (1983). A normative model of work team effectiveness. Technical Report, $n^{\circ} 2$ Research Program on Group Effectiveness, Yale School of Organization and Management HACKMAN, J. R. (1987). The Design of Work Teams. In J.W. Lorsch (ed.). Handbook of Organizo tional Behaviour. Englewood Cliffs, N. J.: Prentice Hall.

HACKMAN, J. R. (1990). Groups That Work and Those That Don't. San Francisco: Jossey-Bass.

HACKMAN, J. R. \& MORRIS, C. G. (1975). Group Tasks, Group Interaction Process and Group Performance Effectiveness: a review and Proposed Integration, In L. Berkowitz (ed.). Advan ces in Experimental Social Psychology, 8, New York Academic Press.

HACKMAN, J. R. \& OLDHAM, G. R. (1980). Work redesign. Reading MA:Addison-Wesley.

HANNAN, M.T. \& FREEMAN. J.H. (1977), Obstacles to the comparative study of organizational effectiveness. In P.S. Goodmaan \& J. M. Pennings (eds). New Perspectives on Organizational Effectiveness. San Francisco: Jossey-Bass.

MAYO, E. (1932). Human Problems of an Industrial Cvilization, Boston, Harvard School of Business. MIDDLEMIST, R. D. \& HITT, M.A. (1981). Technology as a moderator of the relationship between perceived work environment and subunit effectiveness. Human Relations, 34, (6), 517-532.

MORIN, E., SAVOIE, A. \& BEAUDIN, G., (1994). L'Éfficocité de l'Organization - Théories, Représentations et Mesures. Montréal: Gaëtan Morin Éditeur.

NIEVA, V. F., FLEISHMAN, E. A. \& RIECK, A. (1978). Team Dimensions: Their Identity, Their Measurement and Their Relotionships. Washington D.C.:Advanced Research Resources Organizations.

PFEFFER, J. (1977). Usefulness of the Concept. In P. S. Goodman \& J. M. Pennings (eds.). New Perspectives on Organizational Effectiveness. San Francisco: Jossey-Bass.

QUINN, R. E. \& ROHRBAUGH. J. (1983). A Spatial Model of Effectiveness Criteria: Towards a Competing Values Approach to Organizational Analysis. Management Science, 29, 363-377.

SAVOIE, A. \& BEAUDIN. G. (1995). Les Equipes de Travail: que faut-il en connaître?. Psychologie du Travail et des Organizations, I, (2-3), 116-137.

SHEA. G. P. \& GUZZO, R A. (1987). Groups as Human Resources, Research in Personnel and Human Resources Management, 5. 323-356.

STEINER I. D. (1972). Group Processes and Productivity. New York Academic Press.

SUNDSTROM, E. DEMEUSE, K. P. \& FUTRELL. D (1990). Work Teams: applications and effectiveness. American Psychologist,45, 120.133.

TANNENBAUM, S. I.. BEARD, R L. \& SALAS, E. (1992). Team Building and its influence on team effectiveness: an examination of conceptual and empirical developments. In K. Kelley (ed). Issues theory and research in Industrial/Organizational Psychology.Amsterdam: Elsevier.

TRIST, E. L \& BAMFORTH, K.W. (1951). Some social and psychological consequences of the longwall method of coal-getting. Human Relations, 4, 3-38.

TZINER A. \& VARDI.Y. (1982). Effects of comment style and group cohesiveness of self-selected tank crews. Journal of Applied Psychology. 67 (6), 769-775.

WEICK, K. E. (1977). Re-punctuating the Problem. in P. S. Goodman \& J. M. Pennings (eds.), New Perspectives on Organizational Effectiveness. San Francisco: Jossey-Bass. 
(Página deixada propositadamente em branco) 
Série

Investigação

Coimbra

Imprensa da Universidade

2000 